\title{
Maxillomandibular Advancement and Upper Airway Stimulation: Extrapharyngeal Surgery for Obstructive Sleep Apnea
}

\author{
Myeong Sang Yu ${ }^{1,2}$ (1) $\cdot$ Badr Ibrahim ${ }^{1} \cdot$ Robert Wayne Riley ${ }^{1} \cdot$ Stanley Yung-Chuan Liu ${ }^{1}$ (C) \\ ${ }^{1}$ Division of Sleep Surgery, Department of Otolaryngology-Head and Neck Surgery, Stanford Hospital and Clinics, Stanford, CA, USA; \\ ${ }^{2}$ Department of Otorhinolaryngology-Head and Neck Surgery, Asan Medical Center, University of Ulsan College of Medicine, Seoul, Korea
}

\begin{abstract}
There are many ways to categorize surgery for obstructive sleep apnea (OSA), one of which is to distinguish between intrapharyngeal and extrapharyngeal procedures. While the general otolaryngologist treating OSA is familiar with intrapharyngeal procedures, such as uvulopalatopharyngoplasty and tongue base reduction, extrapharyngeal sleep operations such as maxillomandibular advancement (MMA) and upper airway stimulation (UAS) have evolved rapidly in the recent decade and deserve a dedicated review. MMA and UAS have both shown predictable high success rates with low morbidity. Each approach has unique strengths and limitations, and for the most complex of OSA patients, the two in combination complement each other. Extrapharyngeal airway operations are critical for achieving favorable outcomes for sleep surgeons.
\end{abstract}

Keywords: Obstructive Sleep Apnea; Sleep Surgery; Maxillomandibular Advancement; Facial Skeletal Surgery; Hypoglossal Nerve Stimulation; Upper Airway Stimulation

\section{INTRODUCTION}

Globally, 936 million adults aged 30 to 69 years have been diagnosed with obstructive sleep apnea (OSA), of whom 425 million have moderate to severe disease [1]. OSA affects approximately $5 \%$ to $10 \%$ of the U.S. adult population [2], and its prevalence ranges from $3.7 \%$ to $27.0 \%$ in the Asian population [3]. OSA and its comorbidities significantly affect patients' quality of life. It is a significant risk factor for hypertension, type II diabetes, stroke, congestive heart failure, coronary artery disease, and premature death [4]. Furthermore, it is associated with poor sleep quality, daytime sleepiness, fatigue, and cognitive dysfunction. Nevertheless, only $40 \%$ of patients with OSA are diagnosed [5,6], of whom a large proportion are not optimally treated [2].

Continuous positive airway pressure (CPAP) is regarded as

\footnotetext{
- Received March 16, 2020

Revised April 12, 2020

Accepted April 16, 2020

- Corresponding author: Stanley Yung-Chuan Liu

Division of Sleep Surgery, Department of Otolaryngology-Head and Neck Surgery, Stanford University School of Medicine, 801 Welch Road, Stanford, CA 94304, USA

Tel: +1-650-724-3336, Fax: +1-650-724-3336

E-mail: ycliu@stanford.edu
}

the first-line treatment in many parts of the world. Adherence remains a challenge, as $46 \%$ to $83 \%$ of patients do not tolerate the therapy long-term [7]. Other nonsurgical therapies include the use of an oral appliance or tongue-retaining device, positional therapy, and medical weight management. Surgeons also play a role in OSA management [8].

There are many ways to categorize surgery for OSA, one of which is to distinguish between intrapharyngeal and extrapharyngeal procedures. The Powell and Riley Stanford algorithm incorporates both; intrapharyngeal procedures include soft palate and tongue base procedures, and extrapharyngeal procedures include genioglossus and maxillomandibular advancement (MMA). The combination of both types of surgical treatment yielded more predictable results than intrapharyngeal surgery alone [9,10]. Recently, Liu et al. [11] revised the Stanford algorithm to incorporate contemporary skeletal techniques and upper airway stimulation (UAS), also known as hypoglossal nerve stimulation. While the general otolaryngologist treating OSA is familiar with intrapharyngeal procedures, which include uvulopalatopharyngoplasty (UPPP) and tongue base reduction, extrapharyngeal sleep operations (MMA and UAS) have evolved rapidly in the recent decade and deserve a dedicated review.

Copyright $\odot 2020$ by Korean Society of Otorhinolaryngology-Head and Neck Surgery

This is an open-access article distributed under the terms of the Creative Commons Attribution Non-Commercial License (https://creativecommons.org/licenses/by-nc/4.0)

which permits unrestricted non-commercial use, distribution, and reproduction in any medium, provided the original work is properly cited. 


\section{THE ROLE OF DRUG-INDUCED SLEEP ENDOSCOPY IN EXTRAPHARYNGEAL SURGERY}

A thorough head and neck examination, including sleep apneaspecific examinations, should be performed. The examination findings are interpreted within the context of the patient's general medical and sleep history. These topics are well-covered in numerous texts. Here, the focus is on how drug-induced sedation (or sleep) endoscopy (DISE) has been used within the context of extrapharyngeal surgery.

DISE has been added to the comprehensive decision-making process for sleep surgery in general [12], and for MMA in particular [13-15]. MMA reverses lateral pharyngeal wall collapse and complete concentric collapse (CCC) of the velum under DISE. As lateral pharyngeal wall collapse is difficult to resolve with intrapharyngeal operations [16-18], MMA can be considered in OSA patients with this airway collapse pattern, regardless of their maxillofacial phenotype [19-21].

CCC of the velum during DISE is an exclusion criterion for UAS $[2,18,22]$. While UPPP and tonsillectomy can change the CCC pattern [23], no study has compared the success rate between these CCC-converted candidates and initially non-CCC patients [24]. Nevertheless, Liu et al. [20,25] have shown that $\mathrm{CCC}$ of the velum is readily reversible with tissue-sparing palatopharyngoplasty or MMA and that CCC reversal procedures can be used to establish patients as candidates for UAS, providing support for its use in combination with UAS. Further evaluations establishing the surgical outcomes in CCC-converted subjects are warranted.

\section{CONTEMPORARY MAXILLOMANDIBULAR ADVANCEMENT}

MMA, a facial skeletal surgical procedure pioneered by Riley and

\section{H I G G H L I G G H T S}

- Maxillomandibular advancement (MMA) and upper airway stimulation (UAS) are extrapharyngeal operations for obstructive sleep apnea (OSA) that have shown high rates of efficacy with stable long-term results.

- The indications for MMA have expanded since the advent of drug-induced sleep endoscopy, as it has been shown to effectively address concentric collapse of the velum and lateral pharyngeal wall collapse.

- Candidacy for UAS can be expanded by a variety of intrapharyngeal and extrapharyngeal procedures that help patients match inclusion criteria.

- Extrapharyngeal surgery lends insights into the mechanisms of maintaining upper airway stability during sleep in OSA patients.
Powell at Stanford University Hospital, is one of the most effective surgical interventions for patients with OSA [20,26-28]. Previously, it was thought that the MMA effectively reduces the apnea-hypopnea index (AHI) by expanding the skeletal frame and allowing more room for the tongue. More recent dynamic examinations have shown that in fact, stability of the velum and lateral pharyngeal wall is most consistently associated with the success of MMA $[14,15]$. Hence, the contribution of MMA to AHI reduction derives both from the stability of upper airway dilator muscles and from the increased intraoral volume for the tongue.

Generally, the indications for MMA are as follows: moderate to severe OSA, and mild OSA in patients who present with a concomitant dentofacial deformity. Other indications include inadequate response to Riley-Powell phase 1 surgery (intrapharyngeal surgery with or without genioglossus advancement) $[11,19$, 21]. Concentric and lateral pharyngeal wall collapse observed on DISE also favor MMA [14,15]. While there is not a strict body mass index (BMI) cutoff, the efficacy of upper airway surgery decreases with increasing BMI. There is also not an AHI cutoff, though it is expected that patients with a higher $\mathrm{AHI}$ will require additional treatment [29].

\section{Preoperative planning}

Virtual surgical planning (VSP) using computed tomography images allows the surgeon to plan and perform MMA virtually $[20,26]$. The plan begins with correction of cant and yaw deformity (if any), followed by rotation and advancement of the maxillomandibular complex (Fig. 1).VSP also allows for a more streamlined and precise splint fabrication process. An adjunctive orthodontic intervention may complement MMA, although MMA is frequently performed without orthodontic decompensation [30,31]. An important distinction should be made between orthognathic surgery and MMA. Orthognathic surgery refers to straightening of the jaws, during which the jaw may be moved in any direction, including posteriorly. This can be unfavorable for the airway [32-35]. MMA does incorporate orthognathic needs, but it prioritizes the airway. The balance of "breathing, bite, and beauty," in that order, is the goal.

\section{Key surgical steps}

Midface degloving followed by an osteotomy through the lateral and medial buttress of the maxilla is performed. A wedge is then removed from the anterior piriform rim to impart a counterclockwise (CCW) rotation (Fig. 2A). Differential anterior impaction is performed with the rotation center at the maxillary buttress. The maxilla is rotated in line with the buttress and the level of the first molar is maintained. CCW decreases the negative esthetic impact of a large maxillary advancement, while maximizing the anterior movement of the mandible [20].

Disjunction of the maxillary posterior wall from the pterygoid plates is performed with a curved osteotome. The nasal septal 

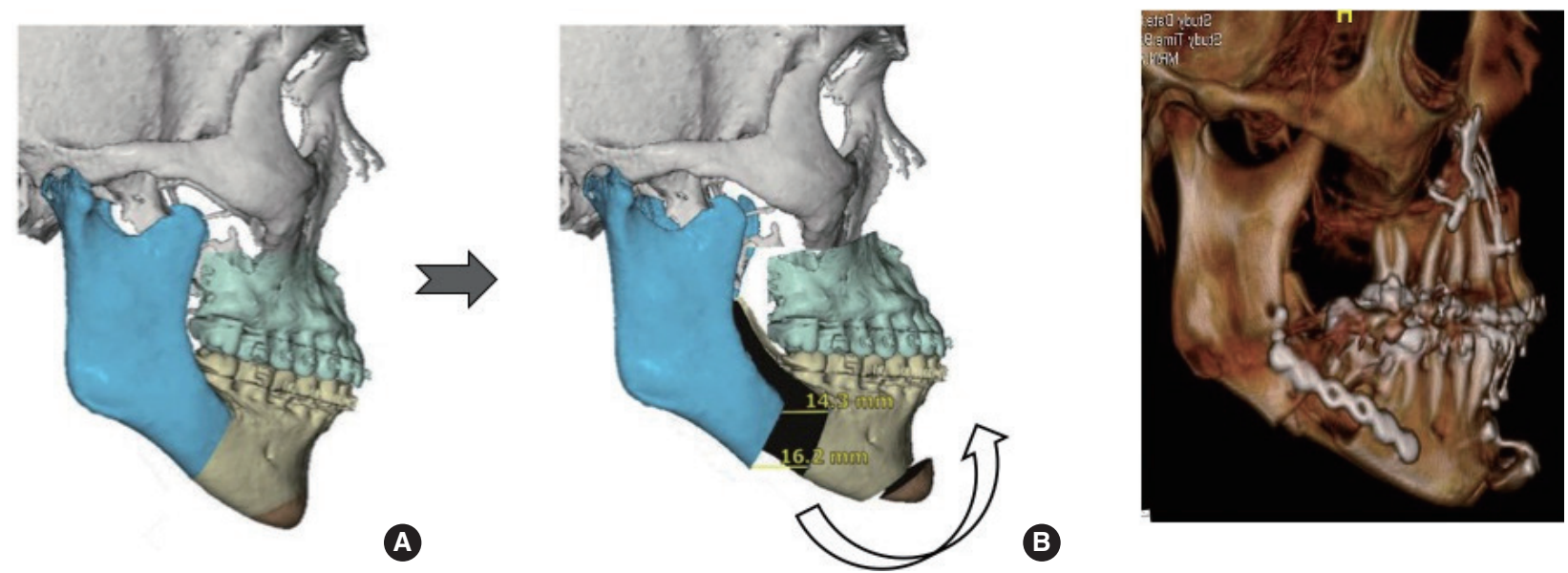

B

C

Fig. 1. Virtual surgical planning (VSP) of maxillomandibular advancement with counterclockwise rotation: preoperative VSP (A), postoperative VSP (B), and postoperative computed tomography scan (C).
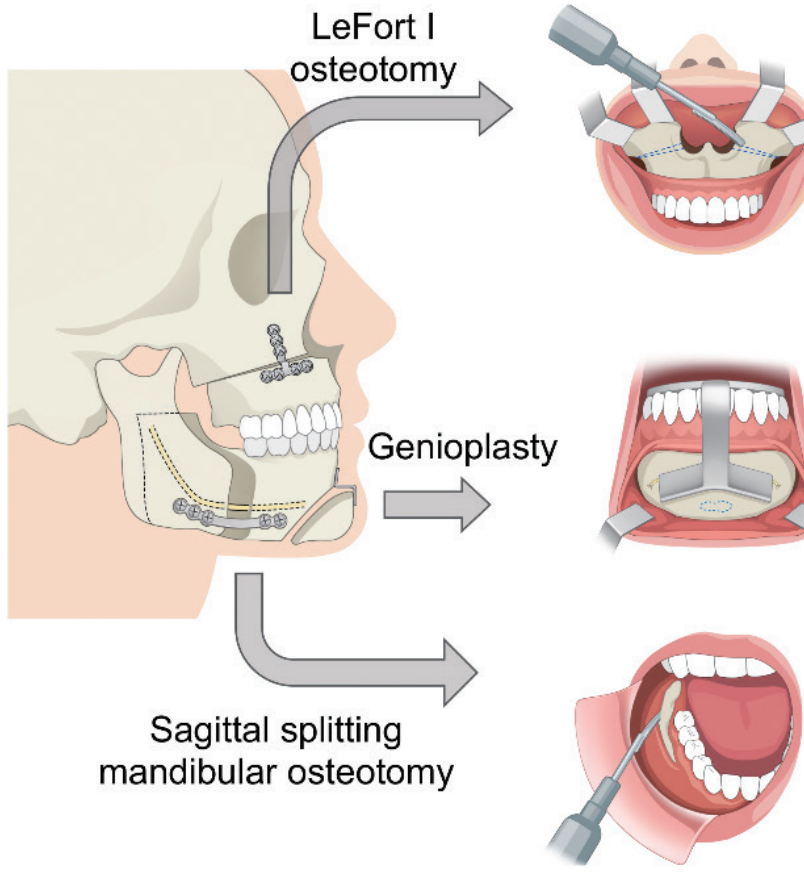

A
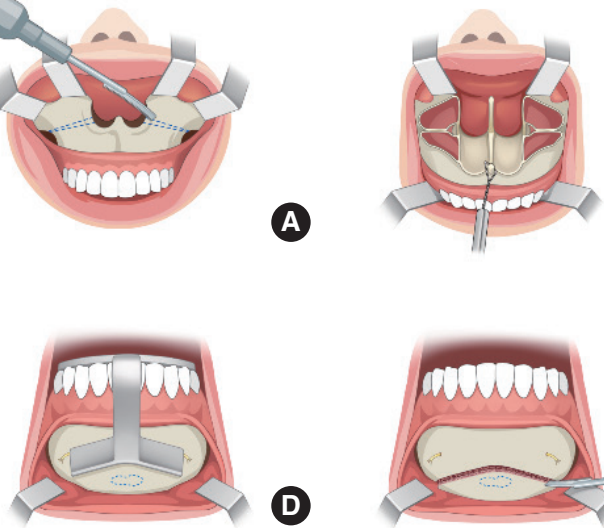

D

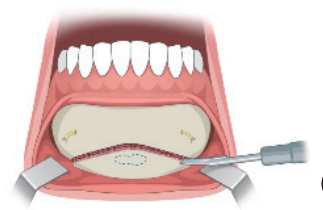

E

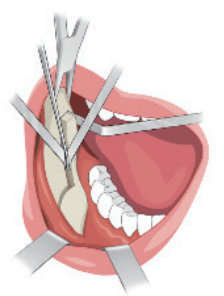

B

G

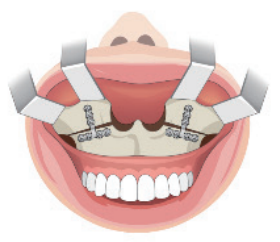

C

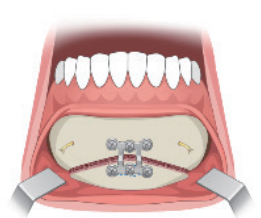

$\boldsymbol{F}$

Fig. 2. Schematic drawing of maxillomandibular advancement. LeFort I osteotomy. (A) Exposure of the piriform aperture and nasal floor. Le Fort I osteotomy and wedges (dotted lines) for counterclockwise rotation. (B) Maxilla mobilization via traction with wire through the anterior nasal spine. (C) Fixation with titanium plates. Virtual surgical planning-guided genioglossus and geniohyoid advancement. (D) The anterior mandible is exposed and the osteotomy guide is designed to capture the genial tubercle (dotted line) while avoiding dental roots and mental nerves. (E) The osteotomy is made using a reciprocating saw. The genial tubercles (dotted line) are preserved. (F) The mobilized graft is moved forward and fixed with a plate. Sagittal split mandibular osteotomy. (G) The mandibular ramus and body are exposed by subperiosteal dissection. $(\mathrm{H})$ The horizontal osteotomy is made through the lingula and the anterior osteotomy split sequentially with three osteotomes. $(\mathrm{I})$ The mandible is advanced, and fixation is performed with positional screws and titanium plates.

attachment to the maxilla is severed and the maxilla is downfractured. The maxilla is mobilized until it can be freely positioned to the planned surgical position confirmed by the intermediate splint, where multiple positions can be fabricated from the VSP process. Traction with wire through the anterior nasal spine and application of lateral forces to the posterior maxillary wall facilitate complete mobilization of the maxilla (Fig. 2B). A septoplasty is performed, followed by widening of the nasal floor and piriform rim. The anterior nasal spine is contoured to minimize overprojection of the nasal tip and widening of the nose. These steps are critical to prevent nasal obstruction after MMA [36]. After fixation of the maxilla with plates, additional 
temporary fixation is made with suspension wires placed from the lateral piriform rim to bone-anchored screws in the maxillary alveolus (Fig. 2C). This is to be used later with the mandibular counterpart for maxillomandibular fixation (MMF), instead of archbars or orthodontic brackets. This increases both stability and accuracy prior to final fixation.

After confirming proper maxillary advancement, bilateral sagittal split ramus osteotomies of the mandible are performed using standard techniques with modifications for older adults with OSA (Fig. 2G) [20]. The proximal and distal fragments of the mandible are gently wedged open with three osteotomes inserted in a sequential sandwiched fashion, instead of forceful use with splitters (Fig. 2H). Age or severity of OSA does not contribute to a favorable split as much as surgical technique. Osteotomy through the lingula and an anterior vertical osteotomy that stops at the midline of the inferior border are associated with ideal mandibular osteotomy for large advancements [37].

After application of the final splint with MMF using bone-anchored wires, the first two bicortical positional screws are applied through the proximal and distal segments using a transfacial trocar. The mandibular plate is then used to span the advancement gap, which usually ranges from 1.2 to $2 \mathrm{~cm}$ (Fig. 2I). The rigid fixation enables a rapid return to function. If indicated, genioglossus advancement is performed before sagittal split osteotomy. The authors preferVSP-guided genial osteotomy to ensure that the genial tubercle is included in the advanced bony segment (Fig. 2D-F), as well as to minimize the risk of injury to the mental nerve or dental roots [38].

\section{Postoperative considerations}

Postoperatively, 6- or 8-ounce guiding elastic bands are applied in a class 2 fashion without a splint. This allows MMA patients to breathe orally when there is significant nasal congestion. Starting the third week after surgery, patients begin consuming a soft diet.

\section{Complications}

Airway compromise represents the most dangerous, although exceedingly rare, complication after MMA. Minimal use of narcotic pain medication is expected postoperatively [20]. Mandibular fracture occurs in $1.0 \%$ of patients, and is commonly associated with concomitant medical comorbidities in older patients [29]. Facial paresthesia is very common and frequently takes a prolonged course to recovery [39]. Hardware is removed in $15 \%$ of MMA patients, with mandibular hardware most commonly removed [20].

\section{Treatment outcomes}

Meta-analyses and long-term follow-up studies have demonstrated that MMA consistently results in a high surgical success rate and a modest cure rate. Holty and Guilleminault [39] performed the largest meta-analysis, examining 22 studies involv- ing 627 patients who underwent MMA for OSA. The study reported a mean AHI decrease from 63.9 to 9.5 events per hour. The authors defined surgical success as achieving at least a $50 \%$ reduction in the AHI, with a final AHI less than 20 events per hour. The surgical success rate was $86.0 \%$ and the cure rate (AHI $<5$ events per hour) was $43.2 \%$. The predictive factors for surgical success included younger age, lower preoperative weight, lower AHI, and a greater degree of maxillary advancement. The major and minor complication rates were $1.0 \%$ and $3.1 \%$, respectively. Another large meta-analysis performed by Zaghi et al. [29], which included 45 studies involving 528 patients, showed that mean postoperative changes in the $\mathrm{AHI}$ and respiratory disturbance index (RDI) after MMA were 47.8 and 44.4 events per hour, respectively. They reported success and cure rates of $85.5 \%$ and $38 \%$, respectively. The most important predictor of surgical cure was a preoperative AHI less than 60 events per hour. Another recent meta-analysis, in which most patients had previously undergone intrapharyngeal surgery, reported that the surgical success rate of MMA using the same criteria was $100 \%$ with respect to AHI and RDI scores [40]. Some studies have shown significant improvements in both the AHI and sleep quality after MMA. Liu et al. [41] reported that sleep architecture normalized after MMA, with an increase in rapid eye movement (REM) sleep and a decrease in wakefulness after sleep onset.

In 40 patients who underwent MMA with an average followup of 4.2 years (range, 1-12 years), 36 patients (90\%) maintained a significant reduction in RDI from 71.2 events per hour to 7.6 events per hour, with improvements in daytime sleepiness [42]. In another study with a mean follow-up of 12.5 years, the surgical success rate was maintained at $100 \%$ in patients less than 45 years old who had a BMI less than $25 \mathrm{~kg} / \mathrm{m}^{2}$ [43].

MMA induces facial changes. Several studies have evaluated patients' attitudes towards esthetic changes after MMA and revealed that the majority of patients were satisfied or neutral about their postoperative appearance [27]. Strategies to control facial soft tissue appearance include suturing the alar base to correct the widening of the nasal base, anterior nasal spine reduction to improve the nasolabial angle, and genioplasty to improve chin prominence [42].

\section{UPPER AIRWAY STIMULATION}

Various systems for UAS have been introduced. Generally, they involve electrical neurostimulation therapy of the hypoglossal nerve, as in the Hypoglossal Nerve Stimulation system (Apnex Medical, St. Paul, MN, USA), the Aura6000 Targeted Hypoglossal Neurostimulation system (ImThera Medical, San Diego, CA, USA), and the Inspire II UAS device (Inspire Medical Systems, Maple Grove, MN, USA) [18]. Of these, the Inspire system has been approved by the Food and Drug Administration for clinical use. It generates a unilateral respiration-synchronized stimula- 
tion of the medial hypoglossal nerve branches and $\mathrm{C} 1$ nerve, leading to tongue stiffening and protrusion during sleep via the genioglossus and geniohyoid muscles. The discussion in this review will focus on the Inspire system.

While UAS mainly affects the dilator muscles in the tongue and expands the retroglossal airway, it can also be effective in patients with isolated retropalatal collapse [44]. This phenomenon of retropalatal opening can be explained by the theory of mechanical coupling, in which anterior movement of the tongue pulls the palatoglossus muscle, affecting the inferior and anterior displacement of the palate [45]. Indications and contraindications for UAS are presented in Table $1[18,22,46]$.

\section{Anatomical considerations}

The hypoglossal nerve (CN XII) innervates both the tongue pro-

Table 1. Indications and contraindications for upper airway stimulation

\begin{tabular}{l}
\hline Indication and contraindication \\
\hline Indication \\
- Moderate to severe OSA: AHI $15-65$ events per hour \\
- PAP failure \\
- Age $>22 \mathrm{yr}$ \\
- BMI $<32 \mathrm{~kg} / \mathrm{m}^{2}$ \\
Contraindication \\
- Central+mixed apnea $>25 \%$ of the total AHI \\
- Complete concentric palatal collapse on DISE \\
- Pregnancy \\
- Preexisting anatomic variants or neurologic disorders \\
- Patients who require MRI
\end{tabular}

OSA, obstructive sleep apnea; AHI, apnea-hypopnea index; PAP, positive airway pressure; BMI, body mass index; DISE, drug-induced sleep endoscopy; MRI, magnetic resonance imaging.

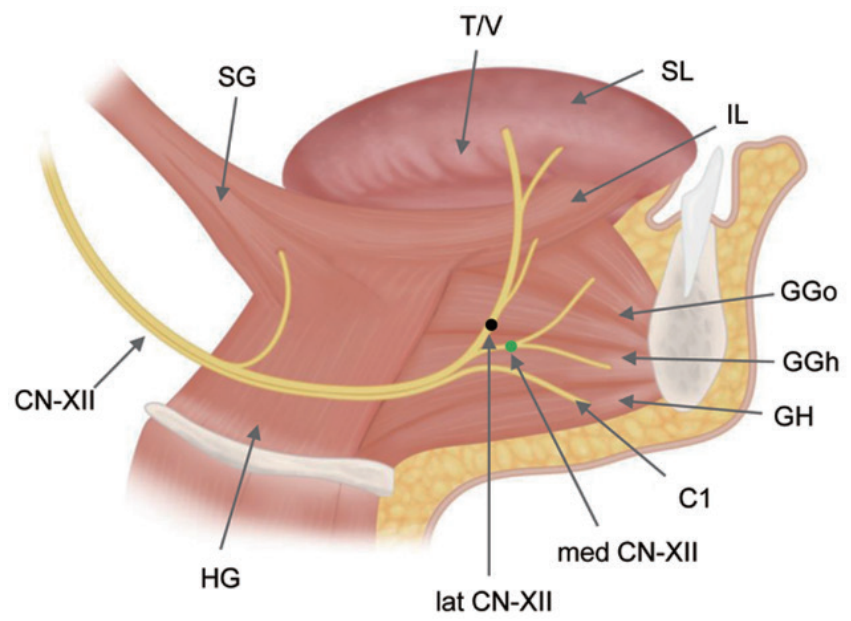

trusor (genioglossus) and tongue retrusor (styloglossus and hyoglossus) muscles through the medial and lateral divisions, respectively. Selective stimulation of the protrusor muscle leads to anterior movement of the tongue, resulting in increased airflow and reduced pharyngeal collapse during sleep [47]. Selective stimulation of the deep and horizontally oriented genioglossus fibers over the superficial and oblique genioglossus fibers results in anterior displacement with curling and stiffening of the tongue, further expanding the upper airway [48]. The first cervical spinal nerve (C1) along with the medial branch of CN XII, innervates the geniohyoid muscle and contributes as an extrinsic protrusor and intrinsic stiffener (Fig. 3). While recommended for inclusion, it has not yet shown additional benefit in OSA treatment [49]. A radiologic study revealed that smaller baseline soft palate volume and greater anterior tongue movement with stimulation improved treatment response to UAS [50].

To achieve maximum airway expansion through anterior displacement of the tongue, the medial division of CN XII and C1 must be included, while avoiding the lateral division of CN XII (Fig. 3) [48,51]. Surgeons need to keep in mind the complex surgical anatomy of CN XII and identify the optimal location for the stimulation cuff in selective UAS.

\section{Key surgical techniques}

The implantation of UAS is performed under general anesthesia. The patient is placed in the supine position, and the patient's neck is extended and turned to the left. A nerve monitoring system (Medtronic, Minneapolis, MN, USA) is applied by introducing probes into the hyoglossus and genioglossus muscles. During surgery, these provide branch-specific feedback to decide which branches of the CN XII to include in the stimulation cuff electrode. After careful placement of the patient, three incisions for

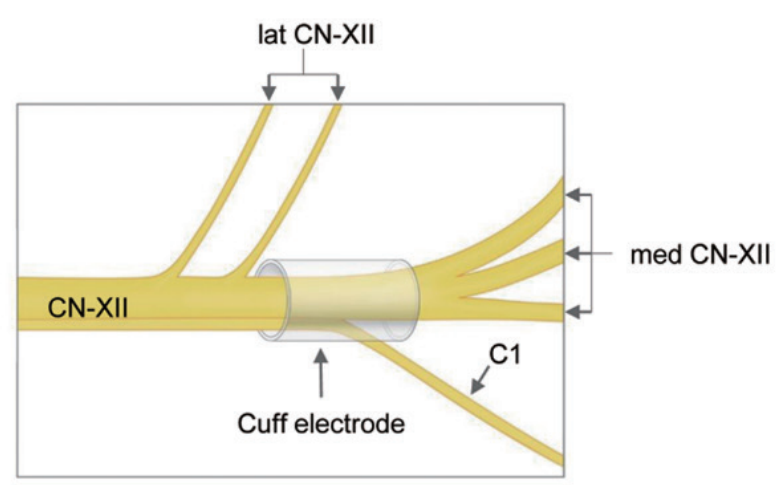

Fig. 3. Tongue muscles and branches of the hypoglossal nerve (CN XII). The medial branches of CN XII (med CN-XII) and first cervical spinal nerve (C1) selectively innervate the primary upper airway dilators: the genioglossus (GG) and geniohyoid (GH) muscles, respectively. The lateral branches of the hypoglossal nerve (lat CN-XII) innervate the retrusor muscles: the hyoglossus (HG) and styloglossus (SG). The ideal placement of the stimulation cuff electrode is distal to the lateral branch of CN XII, thereby including the medial branch and C1 nerve. TN, transverse and vertical; SL, superior longitudinal; IL, inferior longitudinal; GGo, genioglossus oblique; GGh, genioglossus horizontal. 
the stimulation lead (neck), implantable pulse generator (IPG) pocket (superior chest), and sensing lead (inferolateral chest) are made (Fig. 4).

The first incision in the submandibular region, one finger width below the mandible, is used to place the stimulation lead. After identifying the main trunk of CN XII, stimulation of the various branches of CN XII allows the surgeon to exclude branches producing a stimulation at the hyoglossus and to include branches producing a stimulation of the genioglossus and geniohyoid. The nerve stimulator cuff is placed around the selected inclusion branches of $\mathrm{CN}$ XII and anchored to the anterior belly of the digastric muscle (Fig. 4A).

The IPG pocket incision is made in the right superior chest superficial to the fascia of the pectoralis muscle. This pocket should be approximately $5 \mathrm{~cm}$ wide, corresponding to the dimensions of the IPG, and placed to avoid hindering arm mobility (Fig. 4B). An inferolateral chest incision is made in the fifth or sixth intercostal space to place the sensing lead between the internal and external intercostal muscles, with care taken not violate the pleural space (Fig. 4C) [22].

Finally, the stimulation lead and sensing lead are tunneled to the IPG pocket and the leads are plugged into the IPG. Once assembled, the functioning of the system is tested at various stimulation thresholds. Unhindered protrusion of a stiffened tongue is confirmed as well as the absence of retraction. The sensing lead function is verified by assessing the sensor waveform.
Patients are scheduled for a postoperative evaluation at 1 week. Device activation is performed 1 month after surgery, and patients are instructed to start using their device and increase the voltage gradually over the following weeks. This serves as a habituation period. Titration polysomnography is performed 2 months after surgery. During the titration sleep study, much like CPAP titration, the voltage is incrementally increased until respiratory abnormalities disappear.

\section{Treatment outcomes}

The STAR trial found UAS to be successful, with a median $68 \%$ decrease in AHI severity [52]. Recent meta-analyses indicated that UAS is a safe and effective surgical option for selected patients with moderate to severe OSA. Kompelli et al. [53] analyzed 381 patients across 16 studies and reported a mean AHI reduction of 21.1 events per hour ( $95 \%$ confidence interval [CI], 16.9-25.3 events per hour), a mean oxygen desaturation index decrease by 15.0 (95\% CI, 12.7-17.4), a mean Epworth Sleepiness Scale decrease by 5.0 (95\% CI, 4.2-5.8), and a mean Functional Outcomes of Sleep Questionnaire increase by 3.1 (95\% CI, 2.6-3.4). Costantino et al. [54] compared surgical results across UAS devices, and reported mean AHI reductions of 17.5 events per hour (inspire; 95\% CI, 14.98-20.01 events per hour), 24.20 (ImThera; 95\% CI, 11.01-37.39 events per hour), and 20.10 (Apnex; 95\% CI, 10.58-29.62 events per hour) at 12 months after surgery. The surgical success rate was $72.4 \%$ (Inspire), $76.9 \%$

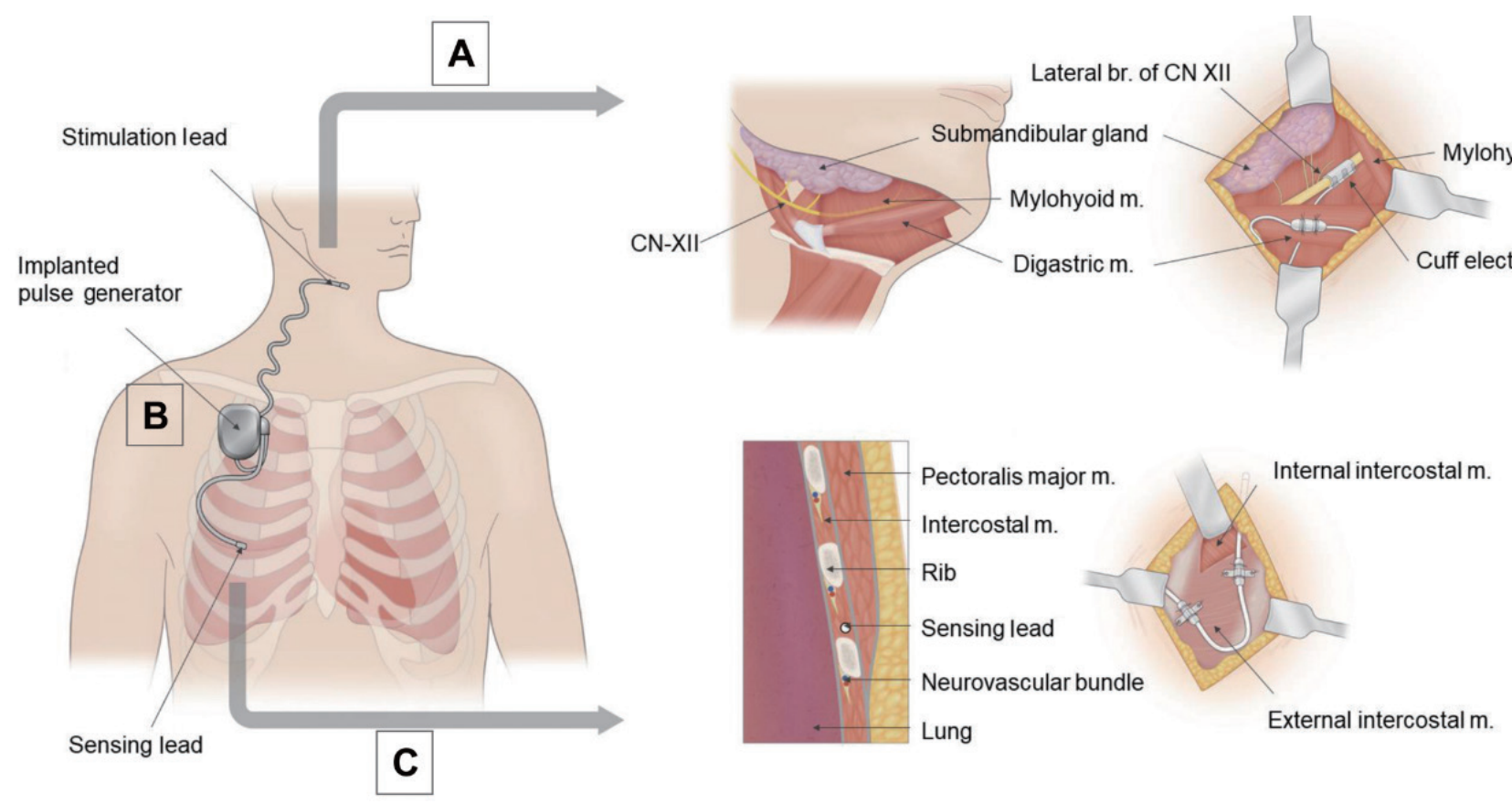

Fig. 4. Hypoglossal nerve stimulation system. (A) Stimulation cuff lead placement. To identify the medial branch (br) of the hypoglossal nerve, the anterior belly of the digastric muscle and mylohyoid muscle $(\mathrm{m})$ are identified and retracted inferiorly and anteriorly, respectively. (B) The implantable pulse generator delivers electric pulses through a stimulation lead to the hypoglossal nerve synchronous with respiratory cycles detected by a sensing lead. (C) Sensing lead placement. The distal end of the sensing lead is placed between internal and external intercostal muscles parallel to the adjacent ribs. 
Table 2. Comparison of the clinical implications of MMA and UAS

\begin{tabular}{lll}
\hline & \multicolumn{1}{c}{ MMA } & UAS \\
\hline Indication & - Dentofacial deformity & - Older patients with preexisting comorbidities \\
& - Phase II surgery as per original Stanford protocol & - AHI: 15-65 events per hour \\
& - Significant lateral pharyngeal wall collapse and concentric collapse of & \\
& the velum on DISE & \\
Strength & - Expands the lateral pharyngeal airway & - Titratable and adjustable \\
& - Possible in patients with very severe AHI (>65 events per hour) and a & - Suitable option for older patients with preexistent \\
& complete circumferential collapse pattern on DISE & comorbidities \\
Limitation & - More invasive intervention & - Short recovery time \\
& - Contraindicated in older patients with preexisting comorbidities & - Contraindicated in patients with complete circumferential \\
& - Longer recovery time & collapse pattern in DISE \\
& & - Incompatibility with MRI \\
\end{tabular}

MMA, maxillomandibular advancement; UAS, upper airway stimulation; AHI, apnea-hypopnea index; DISE, drug-induced sleep endoscopy; MRI, magnetic resonance imaging.

(ImThera), and 55\% (Apnex) at 12 months, and 75\% (Inspire) at a 60-month follow-up.

OSA patients who had difficulties tolerating CPAP also showed a relatively high adherence to UAS. A study including 102 patients revealed that $22.6 \%$ of the patients used UAS therapy for less than 4 hours per night, $77.4 \%$ for 4 hours or more per night, and $55.7 \%$ for more than 6 hours per night [55].

UAS can improve the AHI, as well as several other sleep architecture parameters, among responders. The arousal index and N1 sleep were reduced after UAS, while time spent in N2/N3. There were no significant changes in REM sleep [56]. Comparing the effects of CPAP and UAS on blood pressure and sleepiness symptoms, CPAP yielded greater improvements in blood pressure, while UAS led to greater improvements in sleepiness symptoms [57]. Recent studies have shown that OSA patients with an elevated BMI or those older than 65 years can be successfully treated with UAS $[58,59]$. Complications after UAS include pain $(6.2 \% ; 95 \% \mathrm{CI}, 0.7 \%-16.6 \%)$, tongue abrasion $(11.0 \% ; 1.2-28.7)$, and internal $(3.0 \% ; 0.3-8.4)$ or external device (5.8\%: 0.3-17.4) malfunction [58].

\section{MMA AND UAS: WHEN AND WHY}

MMA and UAS are similar in that they do not involve direct interventions affecting the pharynx, but indirectly and effectively intervene on the pharyngeal airway. In healthy subjects, upper airway dilator muscles prevent upper airway collapse during sleep [18]. Most moderate to severe OSA patients experience airway collapse at multiple sites [60]. Both MMA and UAS are multilevel approaches that increase the size of the retropalatal airway predominantly in the anteroposterior direction and increase the size of the retroglossal airway in anteroposterior and lateral directions [45,61]. MMA fulfills this goal through improved anchorage of airway muscles based on the skeletal position, whereas UAS achieves this goal through dynamic modulation of tongue muscles. In addition, UAS is titratable, and many configurations may be programmed to optimize treatment efficiency $[2,48]$. This adjustability is critical because OSA is a chronic condition that requires continued management long-term (Table 2).

The primary target patient differs between these two procedures. However, MMA and UAS may complement each other. If a patient has an $\mathrm{AHI}$ greater than 65 events per hour, or has concentric and lateral pharyngeal wall collapse during DISE, then MMA can be an option to address both the unfavorable collapse pattern and severe OSA. After MMA, if the AHI remains above 15 events per hour, then UAS can follow. This process frequently achieves a cure. At Stanford, our younger patients with severe OSA usually undergo MMA. In those who are contraindicated for MMA or who elect to undergo UAS, a variety of procedures such as UPPP, genioglossus advancement, tongue base reduction and maxillary expansion can mitigate severe OSA or reverse concentric collapse gain and achieve eligibility for UAS [21,62].

\section{CONCLUSION}

Surgical interventions that treat OSA should address the anatomic abnormality related to the pathophysiology of OSA. MMA and UAS have both shown predictable, relatively high success rates. Each approach has unique strengths and limitations, and for the most complex of OSA patients, the procedures can complement each other. Extrapharyngeal airway operations are critical for achieving favorable outcomes for sleep surgeons.

\section{CONFLICT OF INTEREST}

No potential conflict of interest relevant to this article was reported. 


\section{ACKNOWLEDGMENTS}

The authors would like to thank Hyewon Jung at the Medical Contents Center of Asan Medical Center for support with medical illustrations.

\section{ORCID}

Myeong Sang Yu https://orcid.org/0000-0002-4529-0254

Stanley Yung-Chuan Liu

https://orcid.org/0000-0001-6885-7885

\section{AUTHOR CONTRIBUTIONS}

Conceptualization: SYL, RWR. Data curation: MSY, RWR. Formal analysis: MSY. Methodology: SYL. Writing-original draft: SYL, BI, MSY.Writing-review \& editing: MSY, SYL, BI.

\section{REFERENCES}

1. Benjafield A, Valentine K, Ayas N, Eastwood PR, Heinzer RC, Ip MS. Global prevalence of obstructive sleep apnea in adults: estimation using currently available data [abstract]. In: American Thoracic Society 2018 International Conference; 2018; San Diego, CA, USA. San Diego, CA:American Thoracic Society, 2018;A3962.

2. Soose RJ, Gillespie MB. Upper airway stimulation therapy: a novel approach to managing obstructive sleep apnea. Laryngoscope. 2016 Sep;126 Suppl 7:S5-8.

3. Lam B, Lam DC, Ip MS. Obstructive sleep apnoea in Asia. Int JTuberc Lung Dis. 2007 Jan;11(1):2-11.

4. Salman LA, Shulman R, Cohen JB. Obstructive sleep apnea, hypertension, and cardiovascular risk: epidemiology, pathophysiology, and management. Curr Cardiol Rep. 2020 Jan;22(2):6.

5. Young T, Palta M, Dempsey J, Peppard PE, Nieto FJ, Hla KM. Burden of sleep apnea: rationale, design, and major findings of the Wisconsin Sleep Cohort study.WMJ. 2009 Aug;108(5):246-9.

6. Garrigue S, Pepin JL, Defaye P, Murgatroyd F, Poezevara Y, Clementy $\mathrm{J}$, et al. High prevalence of sleep apnea syndrome in patients with long-term pacing: the European Multicenter Polysomnographic Study. Circulation. 2007 Apr;115(13):1703-9.

7. Kim JH, Kwon MS, Song HM, Lee BJ, Jang YJ, Chung YS. Compliance with positive airway pressure treatment for obstructive sleep apnea. Clin Exp Otorhinolaryngol. 2009 Jun;2(2):90-6.

8. Powell NB. Contemporary surgery for obstructive sleep apnea syndrome. Clin Exp Otorhinolaryngol. 2009 Sep;2(3):107-14.

9. Li KK, Powell NB, Riley RW, Troell RJ, Guilleminault C. Long-term results of maxillomandibular advancement surgery. Sleep Breath. 2000;4(3):137-40.

10. Camacho M, Liu SY, CertalV, Capasso R, Powell NB, Riley RW. Large maxillomandibular advancements for obstructive sleep apnea: an operative technique evolved over 30 years. J Craniomaxillofac Surg. 2015 Sep;43(7):1113-8.

11. Liu SY,Awad M, Riley R, Capasso R. The role of the revised Stanford protocol in today's precision medicine. Sleep Med Clin. 2019 Mar; 14(1):99-107.

12. Awad M, Okland TS, Nekhendzy V. Drug-induced sleep endoscopy.
Atlas Oral Maxillofac Surg Clin North Am. 2019 Mar;27(1):7-10

13. Kastoer C, Op de Beeck S, Dom M, NeirinckxT,Verbraecken J, Braem MJ, et al. Drug-induced sleep endoscopy upper airway collapse patterns and maxillomandibular advancement. Laryngoscope. 2020 Apr;130(4):E268-74.

14. Liu SY, Huon LK, IwasakiT, Yoon A, Riley R, Powell N, et al. Efficacy of maxillomandibular advancement examined with drug-induced sleep endoscopy and computational fluid dynamics airflow modeling. Otolaryngol Head Neck Surg. 2016 Jan;154(1): 189-95.

15. Liu SY, Huon LK, Powell NB, Riley R, Cho HG, Torre C, et al. Lateral pharyngeal wall tension after maxillomandibular advancement for obstructive sleep apnea is a marker for surgical success: observations from drug-induced sleep endoscopy. J Oral Maxillofac Surg. 2015 Aug;73(8):1575-82.

16. Green KK, Kent DT, D’Agostino MA, Hoff PT, Lin HS, Soose RJ, et al. Drug-induced sleep endoscopy and surgical outcomes: a multicenter cohort study. Laryngoscope. 2019 Mar;129(3):761-70.

17. Soares D, Sinawe H, Folbe AJ, Yoo G, Badr S, Rowley JA, et al. Lateral oropharyngeal wall and supraglottic airway collapse associated with failure in sleep apnea surgery. Laryngoscope. 2012 Feb;122(2):473-9.

18. Vanderveken OM, Beyers J, Op de Beeck S, Dieltjens M,Willemen M, Verbraecken JA, et al. Development of a clinical pathway and technical aspects of upper airway stimulation therapy for obstructive sleep apnea. Front Neurosci. 2017 Sep;11:523.

19. Liu SY, Wayne Riley R, Pogrel A, Guilleminault C. Sleep surgery in the era of precision medicine. Atlas Oral Maxillofac Surg Clin North Am. 2019 Mar;27(1):1-5.

20. Liu SY, Awad M, Riley RW. Maxillomandibular advancement: contemporary approach at Stanford. Atlas Oral Maxillofac Surg Clin North Am. 2019 Mar;27(1):29-36.

21. Liu SY, Powell NB, Riley RW.Algorithm for multilevel treatment: the Riley, Powell, and Liu Stanford experience. In: Friedman M, Jacobowitz O, editors. Sleep and apnea snoring. Amsterdam: Elsevier; 2020. p. 104-9.

22. Gupta RJ, Kademani D, Liu SY. Upper airway (hypoglossal nerve) stimulation for treatment of obstructive sleep apnea.Atlas Oral Maxillofac Surg Clin North Am. 2019 Mar;27(1):53-8.

23. Hasselbacher K, Seitz A, Abrams N, Wollenberg B, Steffen A. Complete concentric collapse at the soft palate in sleep endoscopy: what change is possible after UPPP in patients with CPAP failure? Sleep Breath. 2018 Dec;22(4):933-8.

24. Steffen A. What makes the responder to upper airway stimulation in obstructive sleep apnea patients with positive airway pressure failure? JThorac Dis. 2018 Sep;10(Suppl 26):S3131-S4.

25. Liu SY, Hutz MJ, Poomkonsarn S, Chang CP, Awad M, Capasso R. Palatopharyngoplasty resolves concentric collapse in patients ineligible for upper airway stimulation. Laryngoscope. 2020 Feb 28 [Epub]. https://doi.org/10.1002/lary.28595.

26. Boyd SB. Management of obstructive sleep apnea by maxillomandibular advancement. Oral Maxillofac Surg Clin North Am. 2009; 21(4):447-57.

27. Garg RK, Afifi AM, Sanchez R, King TW. Obstructive sleep apnea in adults: the role of upper airway and facial skeletal surgery. Plast Reconstr Surg. 2016 Oct;138(4):889-98.

28. Barrera JE. Skeletal surgery for obstructive sleep apnea. Otolaryngol Clin North Am. 2016 Dec;49(6):1433-47.

29. Zaghi S, Holty JE, Certal V,Abdullatif J, Guilleminault C, Powell NB, et al. Maxillomandibular advancement for treatment of obstructive sleep apnea: a meta-analysis. JAMA Otolaryngol Head Neck Surg. 2016 Jan;142(1):58-66.

30. Prinsell JR. Maxillomandibular advancement surgery in a site-specific treatment approach for obstructive sleep apnea in 50 consecutive patients. Chest. 1999 Dec;116(6):1519-29.

31. Riley RW, Powell NB, Guilleminault C. Maxillary, mandibular, and 
hyoid advancement for treatment of obstructive sleep apnea: a review of 40 patients. J Oral Maxillofac Surg. 1990 Jan;48(1):20-6.

32. Dahy K, Takahashi K, Saito K, Kiso H, Rezk I, Oga T, et al. Gender differences in morphological and functional outcomes after mandibular setback surgery. J Craniomaxillofac Surg. 2018 Jun;46(6):887-92.

33. Shah DH, Kim KB, McQuilling MW, Movahed R, Shah AH, Kim YI. Computational fluid dynamics for the assessment of upper airway changes in skeletal Class III patients treated with mandibular setback surgery. Angle Orthod. 2016 Nov;86(6):976-82.

34. Tan SK, Leung WK, Tang AT, Zwahlen RA. Effects of mandibular setback with or without maxillary advancement osteotomies on pharyngeal airways: an overview of systematic reviews. PLoS One. 2017 Oct;12(10):e0185951.

35. Yang HJ, Jung YE, Kwon IJ, Lee JY, Hwang SJ. Airway changes and prevalence of obstructive sleep apnoea after bimaxillary orthognathic surgery with large mandibular setback. Int J Oral Maxillofac Surg. 2020 Mar;49(3):342-9.

36. Liu SY, Lee PJ,Awad M, Riley RW, Zaghi S. Corrective nasal surgery after maxillomandibular advancement for obstructive sleep apnea: experience from 379 cases. Otolaryngol Head Neck Surg. 2017 Jul; 157(1):156-9.

37. Chen YF, Ko EC, Zaghi S, Yoon A, Williams R, Riley R, et al. Optimizing mandibular sagittal split of large maxillomandibular advancements for obstructive sleep apnea: patient and surgical factors. Clin Oral Investig. 2020 Mar;24(3):1359-67.

38. Liu SY, Huon LK, Zaghi S, Riley R, Torre C. An accurate method of designing and performing individual-specific genioglossus advancement. Otolaryngol Head Neck Surg. 2017;156(1):194-7.

39. Holty JE, Guilleminault C. Maxillomandibular advancement for the treatment of obstructive sleep apnea: a systematic review and metaanalysis. Sleep Med Rev. 2010 Oct;14(5):287-97.

40. John CR, Gandhi S, Sakharia AR, James TT. Maxillomandibular advancement is a successful treatment for obstructive sleep apnoea: a systematic review and meta-analysis. Int J Oral Maxillofac Surg. 2018 Dec;47(12):1561-71.

41. Liu SY, Huon LK, Ruoff C, Riley RW, Strohl KP, Peng Z. Restoration of sleep architecture after maxillomandibular advancement: success beyond the apnea-hypopnea index. Int J Oral Maxillofac Surg. 2017 Dec;46(12):1533-8.

42. Riley RW, Powell NB, Li KK, Troell RJ, Guilleminault C. Surgery and obstructive sleep apnea: long-term clinical outcomes. Otolaryngol Head Neck Surg. 2000 Mar;122(3):415-21.

43. Vigneron A, Tamisier R, Orset E, Pepin JL, Bettega G. Maxillomandibular advancement for obstructive sleep apnea syndrome treatment: long-term results. J Craniomaxillofac Surg. 2017 Feb;45(2):183-91.

44. Mahmoud AF, Thaler ER. Outcomes of hypoglossal nerve upper airway stimulation among patients with isolated retropalatal collapse. Otolaryngol Head Neck Surg. 2019 Jun;160(6):1124-9.

45. Safiruddin F, Vanderveken OM, de Vries N, Maurer JT, Lee K, Ni Q, et al. Effect of upper-airway stimulation for obstructive sleep apnoea on airway dimensions. Eur Respir J. 2015 Jan;45(1):129-38.

46. Doghramji K, Boon M.The role of upper airway stimulation therapy in the multidisciplinary management approach of obstructive sleep apnea in the adult patient. Laryngoscope. 2016 Sep;126 Suppl 7: S9-11.

47. Oliven A, Odeh M, Geitini L, Oliven R, Steinfeld U, Schwartz AR, et al. Effect of coactivation of tongue protrusor and retractor muscles on pharyngeal lumen and airflow in sleep apnea patients. J Appl
Physiol (1985). 2007 Nov;103(5):1662-8.

48. Dedhia RC, Strollo PJ, Soose RJ. Upper airway stimulation for obstructive sleep apnea: past, present, and future. Sleep. 2015 Jun;38(6): 899-906.

49. Kumar AT, Vasconcellos A, Boon M, Huntley C. Inclusion of the first cervical nerve does not influence outcomes in upper airway stimulation for treatment of obstructive sleep apnea. Laryngoscope. 2020 May;130(5):E382-85.

50. Schwab RJ,Wang SH,Verbraecken J,Vanderveken OM,Van de Heyning $\mathrm{P}, \mathrm{Vos} W \mathrm{G}$, et al.Anatomic predictors of response and mechanism of action of upper airway stimulation therapy in patients with obstructive sleep apnea. Sleep. 2018 Apr;41(4).

51. Heiser C, Knopf A, Hofauer B. Surgical anatomy of the hypoglossal nerve: a new classification system for selective upper airway stimulation. Head Neck. 2017 Dec;39(12):2371-80.

52. Strollo PJ Jr, Soose RJ, Maurer JT, deVries N, Cornelius J, Froymovich $\mathrm{O}$, et al. Upper-airway stimulation for obstructive sleep apnea. $\mathrm{N}$ Engl J Med. 2014 Jan;370(2):139-49.

53. Kompelli AR, Ni JS, Nguyen SA, Lentsch EJ, Neskey DM, MeyerTA. The outcomes of hypoglossal nerve stimulation in the management of OSA: a systematic review and meta-analysis. World J Otorhinolaryngol Head Neck Surg. 2018 Sep;5(1):41-8.

54. Costantino A, Rinaldi V, Moffa A, Luccarelli V, Bressi F, Cassano M, et al. Hypoglossal nerve stimulation long-term clinical outcomes: a systematic review and meta-analysis. Sleep Breath. 2020 Jun;24(2): 399-411.

55. Hofauer B, Steffen A, Knopf A, Hasselbacher K, Heiser C.Adherence to upper-airway stimulation in the treatment of OSA. Chest. 2018 Feb;153(2):574-5.

56. Bohorquez D, Mahmoud AF, Yu JL, Thaler ER. Upper airway stimulation therapy and sleep architecture in patients with obstructive sleep apnea. Laryngoscope. 2020 Apr;130(4):1085-9.

57. Walia HK, Thompson NR, Strohl KP, Faulx MD, Waters T, Kominsky A, et al. Upper airway stimulation vs positive airway pressure impact on BP and sleepiness symptoms in OSA. Chest. 2020 Jan;157(1): 173-83.

58. Withrow K, Evans S, Harwick J, Kezirian E, Strollo P. Upper airway stimulation response in older adults with moderate to severe obstructive sleep apnea. Otolaryngol Head Neck Surg. 2019 Oct;161(4): 714-9.

59. Huntley C, Steffen A, Doghramji K, Hofauer B, Heiser C, Boon M. Upper airway stimulation in patients with obstructive sleep apnea and an elevated body mass index: a multi-institutional review. Laryngoscope. 2018 Oct;128(10):2425-8.

60. Caples SM, Rowley JA, Prinsell JR, Pallanch JF, Elamin MB, Katz SG, et al. Surgical modifications of the upper airway for obstructive sleep apnea in adults: a systematic review and meta-analysis. Sleep. 2010 Oct;33(10):1396-407.

61. Abramson Z, Susarla SM, Lawler M, Bouchard C, Troulis M, Kaban LB. Three-dimensional computed tomographic airway analysis of patients with obstructive sleep apnea treated by maxillomandibular advancement. J Oral Maxillofac Surg. 2011 Mar;69(3):677-86.

62. Hong SO, Poomkonsarn S, Millesi G, Liu SY. Upper airway stimulation as an alternative to maxillomandibular advancement for obstructive sleep apnoea in a patient with dentofacial deformity: case report with literature review. Int J Oral Maxillofac Surg. 2019 Dec 21:S0901-5027(19)31275-5. 